\title{
A new approach to the classification of mammographic masses and normal breast tissue
}

\author{
Arnau Oliver, Joan Martí, Robert Martí, Anna Bosch, and Jordi Freixenet \\ Institute of Informatics and Applications \\ University of Girona \\ Campus Montilivi, Ed. P-IV, 17071, Girona \\ \{aoliver, joanm, marly, aboschr, jordif\}@eia.udg.es
}

\begin{abstract}
A new approach to mammographic mass detection is presented in this paper. Although different algorithms have been proposed for such a task, most of them are application dependent. In contrast, our approach makes use of a kindred topic in computer vision adapted to our particular problem. In this sense, we translate the eigenfaces approach for face detection/classification problems to a mass detection. Two different databases were used to show the robustness of the approach. The first one consisted on a set of 160 Regions of Interest (RoIs) extracted from the MIAS database, being 40 of them with confirmed masses and the rest normal tissue. The second set of RoIs was extracted from the DDSM database, and contained 196 RoIs containing masses and 392 with normal, but suspicious regions. Initial results demonstrate the feasibility of using such approach with performances comparable to other algorithms, with the advantage of being a more general, simple and cost-effective approach.
\end{abstract}

\section{Introduction}

Breast cancer is considered a major health problem in western countries. A study developed by the American Cancer Society estimates that, in the United States, between one in eight and one in twelve women will develop breast cancer during their lifetime [1]. Mammography is still the most commonly used method for detecting breast cancer at early stages, a crucial issue for a high survival rate. However, it is well known that radiologists can miss a significant portion of abnormalities, as well as that a large number of abnormalities turn out to be benign after biopsy.

Although the presence of both microcalcifications and masses [4] are clear signs of breast cancer, we focus this work only on the detection of masses. Different algorithms have been proposed in recent years for this specific task. Usually, these algorithms are thought as a two step problem: firstly, an algorithm detects regions with a high probability to be a mass, obtaining Regions of Interest (RoIs), and subsequently, another algorithm is used to assure that the detected RoIs depict a true mass. In this work we are interested in the development of an algorithm belonging to this second class.

In contrast with other algorithms [2,7], our proposal is inspired on a widely known approach used in a parallel topic in computer vision. In this sense, we consider that mass detection and face detection and recognition present some similarities. For instance, in face detection an initial algorithm is used in order to detect a face in an image, while in a second step, other algorithms are used to assign a face to one person. Thus, and using this parallelism, the process of finding RoIs (not covered here) can be approached in a similar way to the face detection problem, whereas the mass detection step can be thought similar to a face recognition problem. A well known method for face recognition is the eigenfaces approach, suggested by Turk and Pentland [8] back in 1991. The way eigenfaces are built and used for recognition presents strong similarities to our detection problem. The eigenfaces approach starts with a database of faces belonging to different persons, and the aim is to classify a new face according to the person it belongs. Actually, a subset of faces of the same person is used into the database in order to cover changes in pose and illumination. Introducing model variability by adding multiple images of the same subject is also an interesting issue for mass detection. In that sense, similar masses belonging to the same type of mass could be introduced to the system in order to account for model mass variation.

In this work, mass detection is regarded as a two-class problem. Therefore our RoI database contains two types of RoIs (following the face recognition simile, contains two people): RoIs containing masses and RoIs of normal tis- 
sue. Different instances for each class are included in the database. Their intra class variability is mainly due to graylevel and texture differences and to the shape and size of the mass or other structures present in the RoI. Again, a parallelism with face detection can also be established related to variations of pose and illumination, respectively. Although this initial paper deals only with the two-class problem (mass or not mass), current work focuses in the extension to more classes (with the aim of recognising different types of masses or other mammographic lesions).

\section{Eigenfaces}

The eigenfaces solution for face recognition was proposed by Turk and Pentland [8], and it is based on the use of the Karhunen-Loeve transform in order to find the vectors that best account for the distribution of face images (forming the face subspace) within the entire image space. Following we explain in more detail this algorithm.

The algorithm begins with a database of $M$ images corresponding to the faces of known individuals. The database contains a set of images for each person including variations of pose and light. Each image has width $w$ and height $h$, and is represented by a (column) vector $x_{k}$ of length $N=w \times h$ containing its gray-level values. The total scatter matrix $\mathrm{S}$ (the covariance matrix) is then calculated as:

$$
S=\sum_{k=1}^{M}\left(x_{k}-\mu\right)\left(x_{k}-\mu\right)^{t}
$$

where $\mu=\frac{1}{M} \sum_{k=1}^{M} x_{k}$ is the (column vector) mean of all face samples. Using the Karhunen-Loeve transform is possible to obtain the face space $W_{p c a}$, which is the subspace that maximises:

$$
W_{\text {pca }}=\arg \max _{W}\left|W^{t} S_{t} W\right|
$$

With such approach, the usefulness of the different eigenvectors to characterise the variation among the images is ranked by the value of the corresponding eigenvalue. Hence, it is possible to reduce the dimensionality of the problem in only a few set of eigenvectors, which are the so called eigenfaces. Thus, the eigenfaces span the face subspace of the original image space, and each face image can be transformed into this space by using them. The result of this transformation is a vector of weights describing the contribution of each eigenface in representing the corresponding input image.

Finally, a model of each face is constructed by doing the above transformation for each face in the database. Thus, when a new face has to be tested, it will be classified as belonging to the most similar class. The similarity is calculated using the Nearest Neighbour algorithm with the Euclidean distance [8].

\section{Adapting to mass detection}

As mentioned earlier, in the eigenfaces approach a parallelism can be established between face images of the same person and mass images. Therefore, by introducing different images (or instances) for each class (mass and normal RoI) we are generalising our model in order to account for a larger variation. In mammography one can also talk about variation in illumination and pose. Changes in illumination are related to the number and energy of X-rays that go through the breast and to the internal density of the breast. On the other hand, changes in the pose can be explained as changes in the global mammogram or in the RoI. Changes of pose in the mammogram are related to the different compression suffered by the breast when the mammogram is acquired. Thus, the shape of a mass, as well as the shape of other internal structures, can be different according to the degree of compression of the breast. In addition, and looking at a RoI level, changes in pose can be seen as changes of size and shape of the mass.

However, the transition from face recognition to mass detection is far from trivial, due to the changes in illumination and pose explained above. Namely, the main drawbacks of applying the eigenfaces approach to the detection of masses are the variance of the gray-level range and the multiple size of the RoIs. Note that the size of the RoIs depends on the size of the (possible) mass, and there is a huge range of masse sizes [4]. Lets see in more detail possible solutions to both drawbacks.

Gray-level and texture variation of RoIs are mainly related to the variation of the acquisition parameters (exposure time, X-ray energy) of mammograms obtained at different time intervals and also to the nature of the breast (breast density and thickness). Using a commonly used simplification, these parameters are considered to affect only to the range of the gray-level values of each RoI. Thus, a solution to take these variations into account can be easily computed by equalising the images. We used a uniform distribution model in this work.

On the other hand, and in contrast with face recognition where a database of faces of the same size is available, the size of the RoIs is not always the same. In order to deal with RoIs of the same size, different proposals are considered:

1. To crop the RoIs by using always the same size. Note, however, that although all the RoIs have the same size, the variability in the size of the masses is still present. The problem of using such approach is that pixels which in one RoI are inside the mass, in another RoI can be found in the mass surrounding tissue, as we can see in first row of Figure 1.

2. A second solution is to crop the exact size of the detected RoI and resize it. Using this approach, pixels 

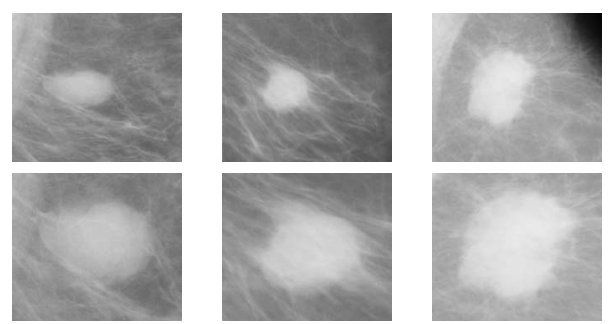

Figure 1. Three different Rols containing clear masses. The first row shows the Rols with the size of the biggest Rol. The second row shows the resized Rols.

will usually have the same behaviour. The drawback of such approach is that texture, which is related to the spatial distribution of the pixels, will be modified. Moreover, the shape of the lesion will also be affected. This solution is shown in second row of Figure 1.

3. Finally, there is another option, which is to cluster the database of RoIs in different groups according to their size. Using this option, the variability of sizes of the masses in each cluster is reduced. Thus, when a new RoI is detected, depending on their size, the corresponding cluster will be used. An illustration of the large variability in the size of the lesions is shown in Figure 2. This variability is usually correlated with the difficulty of detecting such masses. Even for a human observer, mass detection rates increase along with the size of the lesion. This is more pronounced in the case of the smallest lesions.

\section{Results}

In this section, we present the results obtained using our approach. The evaluation is done using a leave-oneout scheme and Receiver Operating Characteristics (ROC) analysis. In the leave-one-out methodology, each RoI is classified using a model trained with the rest of RoIs, and this procedure is repeated until all RoIs have been used as a query image. The classifier used in this work is a combination of the $\mathrm{C} 4.5$ decision tree and the k-Nearest Neighbour algorithm, and returns a numerical value related to the membership to each class. Thus, varying the threshold of this membership is possible to obtain a ROC analysis [5]. In such analysis, a graphical curve represents the true positive rate as a function of the false positives rate. Moreover, the percentage value under the curve $\left(A_{z}\right)$ is an indication for the overall performance of the observer, and is typically used to analyse the performance of the algorithms.

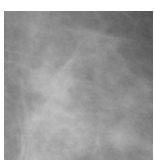

(a)

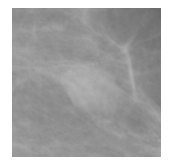

(b)

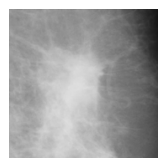

(c)

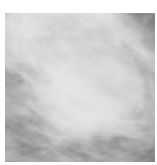

(d)
Figure 2. Four different Rols containing masses, each belonging to a different size group of third proposal, ordered from (a) to (d) from increasing size.

The three different approaches to eliminate the effects of the RoI size were evaluated using the MIAS database [6]. The two first approaches were evaluated using a database of 160 RoIs, 40 depicting a true mass and 120 normal tissue extracted from MIAS database. In the first solution, the RoIs were cropped using the size of the biggest RoI, while in the second approach, all the RoIs were resized in order to have the same size. The obtained $A z$ values were of 0.84 for the first solution and 0.82 for the second one.

The third solution was the classification of the RoIs in different groups according to their size. We have divided the above data into four different groups of 40 RoIs each one. Each group corresponds to the following intervals for mass sizes: < $100 \mathrm{~mm}^{2},(100-180) \mathrm{mm}^{2},(180-360) \mathrm{mm}^{2},>$ $360 \mathrm{~mm}^{2}$. In each interval there were, respectively, 7,11 , 12 and 7 masses, while the rest of RoIs represent normal tissue. We discarded three masses which were much larger than the rest.

The overall performance of this approach was of 0.92 , which is the highest comparing the three solutions. First row of Table 1 shows a detailed comparison of the performance for each group. Clearly, the presented approach is more suitable for larger masses than for small ones. This is due to the fact that larger masses have a larger variation in gray-level contrast with respect to their surrounding tissue than small masses, which are usually more subtle, as it can be seen in Figure 2.

In order to show the robustness of our approach, the last approach was also tested using the DDDM database [3]. We manually extracted 588 RoIs of DDSM database, 196 depicting a true mass and the rest 392 being normal, but suspicious tissue. Four groups were selected according to the size of the lesion: $<120 \mathrm{~mm}^{2},(120-190) \mathrm{mm}^{2},(190-$ 270) $\mathrm{mm}^{2},>270 \mathrm{~mm}^{2}$, and the number of masses at each group were, respectively, 37, 57, 69, and 33. A relation of two RoIs of normal tissue for each RoI mass were used in this experiment.

The overall performance obtained using the third approach was of 0.83 . Second row of Table 1 shows again a comparison of the performance of the method for each size 
Table 1. $A z$ results for the classification of masses using the third approach detailed for each mass group and both databases.

\begin{tabular}{|c|c|c|c|c|}
\cline { 2 - 5 } \multicolumn{1}{c|}{} & $A z(1)$ & $A z(2)$ & $A z(3)$ & $A z(4)$ \\
\hline MIAS & 0.86 & 0.92 & 0.90 & 0.99 \\
\hline DDSM & 0.82 & 0.84 & 0.79 & 0.90 \\
\hline
\end{tabular}

group. Note that although the results are similar for each group, a slightly increase of the performance is shown as the size of the masses increases. Comparing the results of both databases, performance obtained with MIAS are better than the obtained with DDSM. This may be related to the different breast tissue of the RoIs, as RoIs extracted from the DDSM database are denser than the RoIs extracted from the MIAS one. However, this will need further investigation.

The estimated performance of our approach compares favourably with published results from recent approaches $[2,7]$. Note however, that direct comparison is not feasible since the results were obtained from different databases, and also using a different ratio between the number of masses and the number of normal tissue. Further studies are needed to evaluate our approach with other datasets and larger population of screened women.

Figure 3 shows the 9 images constructed by using the eight first eigenvectors of third group of RoIs. As we can see, each image contributes with different information to the system. For instance, the first image (thus, the first eigenvalue) represents the main variation in the gray-level transition going from top-left to down-right. The second one is due to the variation of the gray-level values from the outside and the inside of the image, and thus successively. Note also, that this second eigenvector is related to the nopresence of masses, as well as eigenvectors 6 and 7 are related to their presence.

\section{Conclusions and Further Work}

We have presented a new approach to mass detection based on the adaption of a known face classification technique, the eigenfaces approach. Two main drawbacks were detected when exporting this technique: the gray-level variance and the different size of the RoIs. The former was resolved by using a common equalisation approach, while for the latter three different proposals were used. The performance of all approaches was calculated by using a leaveone-out methodology and ROC analysis. The performance of the three approaches was different, with the latter obtaining the best results $(A z=0.92)$. Moreover, we tested this last approach using another database and higher number of RoIs, obtaining slightly worst performance $(A z=0.83)$.

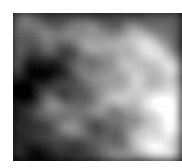

(1)

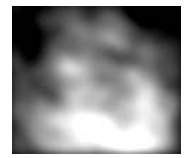

(5)

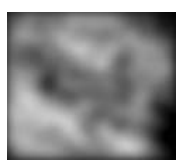

(2)

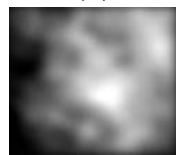

(6)

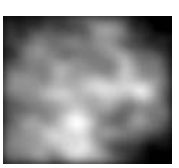

(3)

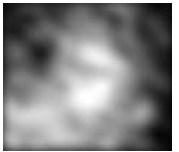

(7)

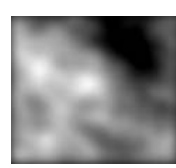

(4)

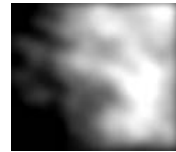

(8)
Figure 3. The first eight eigenimages found in the third group of Rols for the third solution.

We consider these results promising, although a larger database of RoIs is needed in order to assess clinically the benefits of our approach.

Further work will be focused in two ways: firstly, the extension of this paper to a larger database of RoIs, and secondly, to expand the model in order to detect other kind of mammographic lesions, like microcalcifications or architectural distortions, as well as for the classification of different types of masses. This will be related to the extension of the problem to more than two classes.

\section{Acknowledgments}

The authors would like to thank Dr Danica Kragic for her helpful discussions and advice. This work was partially supported by MEC grant nb. TIN2005-08792-C03-01.

\section{References}

[1] American Cancer Society. Breast cancer: facts and figures. 2003-04. ACS, 2003.

[2] Y. Chang, L. Hardesty, et al. Knowledge-based computeraided detection of masses on digitized mammograms: A preliminary assessment. Med. Phys, 28(4):455-461, 2001.

[3] M. Heath, K. Bowyer, et al. The Digital Database for Screening Mammography. In Int. Work. on Dig. Mammography, pages 212-218, 2000.

[4] D. Kopans. Breast Imaging. Lippincott-Raven, Philadelphia, 1998.

[5] C. Metz. Evaluation of digital mammography by ROC analysis. In Int. Work. on Dig. Mammography, pages 61-68, 1996.

[6] J. Suckling, J. Parker, et al. The Mammographic Image Analysis Society digital mammogram database. In Int. Work. on Dig. Mammography, pages 211-221, 1994.

[7] G. Tourassi, R. Vargas-Vorecek, D. Catarious, and C. Floyd. Computer-assisted detection of mammographic masses: A template matching scheme based on mutual information. Med. Phys, 30(8):2123-2130, 2003.

[8] M. Turk and A. Pentland. Eigenfaces for recognition. J. Cogn. Neuroscience, 3(1), 1991. 